This is an author produced version of a paper published in European Journal of Plant pathology. This paper has been peer-reviewed and is proof-corrected, but does not include the publisher pagination.

Citation for the published paper:

Nguyen, Phuong and Vinnere Pettersson, Olga and Olsson, Peter and Liljeroth, Erland. (2010). Identification of Colletotrichum species associated with anthracnose disease of coffee in Vietnam. European

Journal Plant Pathology. Volume: 127 Number: 1, pp 73-87. http://dx.doi.org/10.1007/s10658-009-9573-5

Access to the published version may require journal subscription.

Published with permission from: Springer

The original publication is available at www.springerlink.com

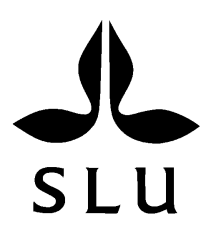

Epsilon Open Archive http://epsilon.slu.se 


\section{Identification of Colletotrichum species associated with anthracnose disease of}

coffee in Vietnam

T.H.P. Nguyen ${ }^{* 1,4}$, O. Vinnere Pettersson ${ }^{2}$, P. Olsson ${ }^{3}$ and E. Liljeroth ${ }^{1}$

1 Department of Plant Protection Biology, Swedish University of Agricultural Sciences, PO box 102, SE-230 53 Alnarp, Sweden

${ }^{2}$ Department of Microbiology, Swedish University of Agricultural Sciences, PO box 7025, SE-750 07 Uppsala, Sweden

${ }^{3}$ Department of Chemistry, Division of Pure and Applied Biochemistry, PO box 124, SE-221 00 Lund, Sweden

4 Department of Plant Molecular Pathology and Biological Control, Institute of Agricultural Genetics and Biotechnology (AGBI), Pham Van Dong Street, Tuliem, Hanoi, Vietnam

*Corresponding author: Phuong.Nguyen@ltj.slu.se

Keywords: Coffee berry anthracnose, Colletotrichum gloeosporioides, pathogenicity test, phylogenetic analysis, tartrate metabolism

\section{Abstract:}

Colletotrichum gloeosporioides, C. acutatum, C. capsici and C. boninense associated with anthracnose disease on coffee (Coffea spp.) in Vietnam were identified based on morphology and DNA analysis. Phylogenetic analysis of DNA sequences from the internal transcribed spacer region of nuclear rDNA and a portion of mitochondrial 
small subunit rRNA were concordant and allowed good separation of the taxa. We found several Colletotrichum isolates of unknown species and their taxonomic position remains unresolved. The majority of Vietnamese isolates belonged to $C$. gloeosporioides and they grouped together with the coffee berry disease (CBD) fungus, C. kahawae. However, C. kahawae could be distinguished from the Vietnamese C. gloeosporioides isolates based on ammonium tartrate utilization, growth rate and pathogenictity. C. gloeosporioides isolates were more pathogenic on detached green berries than isolates of the other species, i.e. C. acutatum, C capsici and C. boninense. Some of the C. gloeosporioides isolates produced slightly sunken lesion on green berries resembling CBD symptoms but it did not destroy the bean. We did not find any evidence of the presence of $C$. kahawae in Vietnam. 


\section{Introduction}

The genus Colletotrichum consists of several economically important plant pathogenic fungi, occurring predominantly in tropical and subtropical regions on a wide range of crops (Waller. 1992). Single species of Colletotrichum can infect multiple hosts. For example, C. gloeosporioides Penz, is pathogenic on citrus, avocado, papaya, and other crops. Also, multiple species of Colletotrichum can be jointly associated with anthracnose on a single host. For example, several Colletotrichum species can cause disease on coffee (Freeman. 2000). C. kahawae Waller, causes coffee berry disease (CBD), which can cause yield losses up to 80\% (Varzea et al. 2002; Chen et al. 2005) in Africa. C. gloeosporioides and C. acutatum Simmonds also occur on coffee and may cause minor disease on ripening berries (Masaba and Waller 1992). However, it has also been reported that $C$. gloeosporioides can over grow C. kahawae and enhance the CBD infection process when the berries become mature or under conditions of environmental stress (Chen et al. 2003). C. kahawae can be distinguished from C. gloeosporioides by its inability to utilize citric acid and ammonium tartrate as sole carbon sources, by its high pathogenicity on coffee and by molecular markers as AFLP and VNTR-PCR (Waller et al. 1993; Bridge et al. 2008). Since C. kahawae causes a specific disease on coffee, there are practical reasons for considering this as a distinct taxon (Bridge et al. 2008). However, according to Cannon et al. (2008), C. kahawae is closely related to the $C$. gloeosporioides complex due to morphological and genetic similarities. 
In Vietnam, anthracnose diseases on Coffea spp. have occurred during recent years. The highest infection rate on coffee berries usually appears in September during berry development and ripening and up to $50 \%$ of the berries on C. arabica can be affected (Tran et al. 1998). Symptoms of anthracnose somewhat resemble those of the CBD, i.e. slightly sunken or brown blight lesions occur on green and ripening berries that can interfere with pulping during seed processing and also be responsible for shedding of premature berries (Pinkert. 2004; Nguyen et al. 2009). In our previous study (2009) we found that certain Vietnamese isolates of C. gloeosporioides were pathogenic on green berries and revealed a high level of genetic variation in the $C$. gloeosporioides populations. C. acutatum and C. capsici or C. dematium have also been found on coffee roots and leaves in Vietnam (unpublished).

Traditionally, identification of Colletotrichum species has primarily relied on morphological characters such as colony color, size and shape of the conidia, growth rate, presence or absence of setae, and existence of the teleomorph, Glomerella (Smith \& Black. 1990). However, the classical identification of Colletotrichum species has encountered some limitations due to the plasticity of morphological traits under environmental impact (Sutton. 1992). In addition, differentiation between Colletotrichum species based on host range can be unreliable, since taxa such as $C$. gloeosporioides, C. dematium, C. acutatum and C. graminicola infect a broad range of host plants (Sutton. 1992). 
Molecular approaches have been successfully used in addition to morphological studies in identification of Colletotrichum species. Sequencing analysis of the ribosomal DNA (rDNA), a fragment of mitochondrial small subunit rRNA gene (mtSSU) and the ß-tubulin gene have been used for species and subspecies delineation of Colletotrichum (Sreenivasaprasad et al. 1993; Vinnere et al. 2002). Species specific primers have been designed from those regions and used for species identification (Sreenivasaprasad et al. 1996).

There have only been a few studies on the causal agents of anthracnose disease on coffee outside Africa. Recently, C. gloeosporioides and C. acutatum responsible for anthracnose disease on Coffea arabica were found in Papua New Guinea (Kenny et al. 2006). To our knowledge, no careful investigation on which Colletotrichum species that are associated with anthracnose disease on coffee in Vietnam has ever been carried out. Therefore, the aims of this study were to: i) identify species of Colletotrichum that are associated with anthracnose disease on Coffea spp. in Vietnam ii) and determine their pathogenic ability 


\section{Materials and methods}

\section{Fungal isolates}

Forty-six Colletotrichum isolates (Table 1) were obtained from infected berries, leaves and twigs of Coffea spp. from five Vietnamese provinces, Ha Tay, Yen Bai, Son La, Nghe An and Dak Lac (Fig. 1). The majority of collected samples were premature green berries with slightly sunken and dark brown lesions. The diseased samples were surface disinfested with commercial chlorine bleach (ColgatePalmolive AB, Danderyd, Sweden) containing $1.5 \%$ of sodium hypochlorite ( $\mathrm{pH}$ 12.8) for 7-10 min and rinsed three times in sterile distilled water. Isolations were made by placing small pieces taken from the margin between diseased and healthy tissues on potato dextrose agar (PDA) (Difco, France) amended with tetracycline (100 $\mu \mathrm{g} / \mathrm{ml}$ ) (Duchefa, the Netherlands), followed by incubation at room temperature $\left(22 \pm 2^{\circ} \mathrm{C}\right)$. Fungal hyphae growing from the pieces were transferred to fresh PDA.

Isolates of C. kahawae (CBD), C. capsici, C. gloeosporioides, C. acutatum and C. boninense previously identified by reliable sources were used as references (Table 1). The cultures were preserved on 50\% PDA slants at $4{ }^{\circ} \mathrm{C}$ under paraffin oil, as conidial suspensions in $50 \%$ glycerol at $-80{ }^{\circ} \mathrm{C}$ and as mycelial blocks in sterile distilled water at $4{ }^{\circ} \mathrm{C}$.

\section{Morphology and culture characteristics}


Agar plugs from 7-day-old cultures were placed onto fresh PDA medium. Colony and conidial characters were recorded after 6 to 7 days of growth at $25^{\circ} \mathrm{C}$ in darkness. To induce sporulation, some cultures were placed in a 12-h photoperiod under near ultra violet (black) light (Phillips TL20W/08) and/or transferred to potato carrot agar (PCA) or oat meal agar (OMA) (Difco, UK). Samples of conidia were taken and mounted in water. The conidial shape was observed under a light microscope (Leica Microsystems) and photographs were taken with a Leica Digital camera DC 300 (Leica Microsystems). For each isolate, length and width of 25 randomly chosen conidia were measured by using the image analysis program Leica Qwin Standard (Leica Microsystems).

To measure growth rates on PDA after 5 days, plates were inoculated with 5 mmdiameter mycelial plugs (5 mm) excised from the margin of a 7-day-old PDA culture. Cultures were incubated in the darkness at $15,20,25,30$ and $35{ }^{\circ} \mathrm{C}$ with three replicates. The radius was measured at the cardinal points for each plate. The experiment was repeated at 20,25 and $30{ }^{\circ} \mathrm{C}$ with representative isolates, i.e. 413(1) and $606 \mathrm{~b}(1)$

\section{Substrate utilization tests}

Isolates of C. gloeosporioides were tested for their ability to utilize ammonium tartrate as a sole carbon source according to the method described by Waller et al. 
(1993). C. kahawae was included as control since it is known not to be able to metabolize tartrate.

\section{Pathogenicity tests}

Twenty-eight Vietnamese isolates of Colletotrichum were tested for pathogenicity on detached green berries of the Arabica cultivar 'Catimor'. One isolate of the CBDfungus (C. kahawae) was used as a positive control in the tests (Table 3). A modified method based on the description given by Bock (1956) was used. Green berries were first sterilized as above. Ten non-wounded and ten wounded green berries (the surface was pierced 1-2mm deep with a sterile needle prior to inoculation) were used for each replicate. The berries were placed on moistened sterilized sand in a plastic box maintained at high humidity. A droplet of conidial suspension $\left(2 \mathrm{x} \quad 10^{6}\right.$ conidia/ml) or a $5 \mathrm{~mm}$ agar plug of mycelia was placed on the surface of the berries. For the former, the inoculation was repeated after $48 \mathrm{~h}$. Symptoms on coffee berries were observed 11 days after the inoculation. The number of infected berries was counted and the lesion diameter of the infected berries was measured. Three replicates were used for each isolate. The experiment was repeated with some of the

pathogenic C. gloeosporioides isolates, i.e. 119b(1), 702(1),531(7), 606b(4) and SL1a(9) and some representative isolates of the other identified species, i.e. BV14, 413(1) and 606b(1).

\section{DNA extraction}


Five mycelial disks derived from the edge of 7-day-old actively growing colonies on PDA were transferred into a Petri dish with $20 \mathrm{ml}$ glucose yeast extract medium (GYM). The cultures were incubated for 5 days at $25^{\circ} \mathrm{C}$. Mycelia were washed with sterile distilled water, harvested by vacuum filtration through a Munktell filter paper (grade 3) and grinded under liquid nitrogen. DNA was extracted as previously described (Nguyen et al. 2009). The DNA working concentration was standardized by addition of DNase and RNase free water after assessment of DNA concentration and quality by using spectrophotometry (ND-1000, Nanodrop) and 1.4\% (w/v) agarose gel electrophoresis.

\section{Identification by using specific primers}

Col1 and Col2 specific primers, designed from 5.8S-ITS region by Martinez-Culebras et al. (2003), were used in identification of Colletotrichum genus. DNA amplification of all Vietnamese isolates by using these primers was carried out according to the method previously described by Martinez-Culebras et al. (2003).

A species-specific primer located within the ITS1 region (Sreenivasaprasad et al. 1992; 1996; Ureña-Padilla et al. 2002) was used in combination with the conserved universal primer, ITS4 (White et al. 1990) to confirm morphological identification of Colletotrichum isolates. The ITS1 primers were either Cg/fInt1 or CaInt2 that are specific for C. gloeosporioides and C. acutatum, respectively. Amplification was performed in $20 \mu \mathrm{l}$ reaction volume containing 50-100ng of DNA, $10 \mathrm{mM}$ Tris $\mathrm{pH}$ 8.3, $50 \mathrm{mM} \mathrm{KCl}, 1.5 \mathrm{mM} \mathrm{MgCl}_{2}, 200 \mu \mathrm{M}$ dNTP, $1 \mu \mathrm{M}$ of primer and 1 unit of Taq 
polymerase (Sigma-Aldrich, St. Louis, MO, USA). The PCR reaction was carried out in a Gen Amp PCR system 9700 thermal cycler (Applied Biosystems, Foster City, CA, USA), starting with a denaturation step at $95{ }^{\circ} \mathrm{C}$ for $5 \mathrm{~min}$, followed by 34 cycles consisting of denaturation at $94{ }^{\circ} \mathrm{C}$ for $1 \mathrm{~min}$, annealing either at $55{ }^{\circ} \mathrm{C}$ (for CaInt2) or $59{ }^{\circ} \mathrm{C}$ (for $\mathrm{Cg} / \mathrm{fInt} 1$ ) for $1 \mathrm{~min}$ and $2 \mathrm{~min}$ of extension at $72^{\circ} \mathrm{C}$ and final extension step at $72{ }^{\circ} \mathrm{C}$ for $10 \mathrm{~min}$. The amplified products were separated on $1.5 \%$ agarose gels and stained in 0.5xTBE (Tris-Borate-EDTA) buffer with ethidium bromide $(0.5 \mu \mathrm{g} / \mathrm{ml})$.

\section{PCR amplification and DNA sequencing}

The ITS and mtSSU regions were amplified by using the ITS1/ITS4 primers (White et al. 1990) and NMS1/NMS2 primers (Li et al. 1994) respectively. Both regions were amplified by using PfuUltra II fusion HS DNA polymerase according to the manufacturer's instruction (Stratagene, Delaware, CA, USA). The amplified DNA products were purified using Qiagen QIAquick PCR Purification Kit (Qiagen AG, Basel, Switzerland). The purified DNA was sequenced on both strands using the same primers as used in the PCR reaction by MWG Biotech (Ebersberg, Germany). Sequencing results were evaluated using Phred/Phrap (Ewing \& Green. 1998). Stretches of contig sequences having bases showing a continuous quality value equal to or above 30 were accepted and used for further analysis.

\section{Phylogenetic analysis}


The resulting sequences were aligned with ClustalW using the BioEdit software 7.0.5 (Tom Hall, North Carolina State University, Raleigh, NC), followed by manual adjustments where necessary. Parsimony analysis was carried out on the aligned sequences of ITS and mtSSU rDNA separately using PAUP (Phylogenetic Analysis Using Parsimony) 4.0b software (Sinauer Associates Inc., Sunderland, MA). Gaps were treated as missing data with the exceptions for several parsimony-informative

gaps in the ITS data set that were treated as the $5^{\text {th }}$ character. All characters were of type "unordered" and had equal weight. The reliability of tree topologies was evaluated by bootstrap analysis using 1000 replications in heuristic search combining 10 random replicates by stepwise addition. Fusarium redolens with accession number AF008562 and U34507 for the ITS and mtSSU region, respectively, was used as an outgroup.

\section{Statistical analysis}

Differences in conidial sizes and growth rates were investigated with Tukey's multiple range test. Differences in pathogenicity between species, i.e. frequencies of infected berries, were tested with analysis of variance (GLM) on arcsine transformed frequency values according to Zar (1984):

$p^{\prime}=1 / 2 \times \arcsin \sqrt{X /(n+1)}+\arcsin \sqrt{(X+1) /(n+1)}$

Where $p^{\prime}$ is the transformed frequency, $X$ is the number of infected berries and $n$ is the total number of inoculated berries. 
The analyses were carried out with SAS statistical package (SAS Institute Inc., Cary, NC, USA), release 8.2. 


\section{Results}

\section{Morphological characterization}

Mycelia in pure cultures of all Vietnamese isolates were light to dark grey. Some dark grey isolates produced abundant sclerotia. Several isolates, e.g. 606b (1), LD35a (T2) and LD11 (L3), formed Glomerella teleomorphs in pure culture. All isolates were divided into three groups depending on conidial shape. Thirty-nine isolates had cylindrical conidia rounded at one or both ends, five isolates had fusiform conidia,

and finally, conidia of two isolates, BV14 and SL204a, were falcate. The conidia of the Vietnamese Colletotrichum varied considerably in length and width (Table 2).

In addition, several isolates with dark grey color had larger cylindrical conidia than any of previously described species of Colletotrichum, e.g. isolates BMT25 (L3) and LD16 (L2) had conidial sizes ranged 25.4- 40.6 x 8.4-11.7 $\mu \mathrm{m}$.

\section{Substrate utilization}

All Vietnamese Colletotrichum isolates, except 606b (1), LD30a (T4), BMT10 (L2), BMT16 (T3) and NA40a (11), were able to use ammonium tartrate as a sole carbon source (Table 1). All three reference CBD isolates (C. kahawae) and one of the reference isolates of $C$. boninense, (isolate MAFF 306094) could not metabolize ammonium tartrate.

\section{Fungal growth rate}


The mycelial growth rates at different temperatures varied greatly among the studied Colletotrichum isolates. All Vietnamese isolates had relatively slow growth at $15^{\circ} \mathrm{C}$. Most isolates that belonged to the group with cylindrical conidia grew rapidly at both 25 and $30{ }^{\circ} \mathrm{C}$, on average $5.4 \mathrm{~mm}^{-1}$ ay $^{-1}$ at $25{ }^{\circ} \mathrm{C}$. However, two of the isolates of unknown species (LD30a (T3) and BMT10 (T2)) had slow growth rate, i.e. 1.9 to $3.15 \mathrm{~mm} \mathrm{day}^{-1}$ at $30{ }^{\circ} \mathrm{C}$. The five isolates with fusiform conidia grew slowly compared to the isolates with cylindrical conidia, had an optimal growth rate around $25{ }^{\circ} \mathrm{C}$ and did not grow at all at $35^{\circ} \mathrm{C}$. The isolates with falcate conidia grew fast at 30 ${ }^{\circ} \mathrm{C}$.

The reference CBD isolates grew fast at 20 and $25{ }^{\circ} \mathrm{C}$, slowly at $30{ }^{\circ} \mathrm{C}$ and did not grow at all at $35{ }^{\circ} \mathrm{C}$ (Fig. 2), whilst the Vietnamese isolates that could not metabolize ammonium tartrate, grew fast at 25 and $30{ }^{\circ} \mathrm{C}$ and grew at $35{ }^{\circ} \mathrm{C}$. When all isolates were compared the growth rate at $30{ }^{\circ} \mathrm{C}$ differed significantly between the CBD isolates and every Vietnamese isolate identified as C. gloeosporioides (Tukey's studentized Range Test, $P=0.05$ ) (Fig. 2)

\section{Identification by using specific primers}

PCR amplification of DNA from the Vietnamese isolates using Col1/Col2 primers, revealed a fragment of approximate 460bp indicating that all isolates belonged to the Colletotrichum genus. 
Five Vietnamese isolates of Colletotrichum with fusiform conidia and the reference isolate of C. acutatum (isolate 397) produced a PCR product of 490bp with CaInt2/ITS4 primers (data not shown).

Twenty-three Vietnamese isolates out of 39 with cylindrical conidia were identified as C. gloeosporioides since these isolates and the reference isolate of $C$. gloeosporioides (PR220) produced a 450bp long fragment after PCR amplification with the CgfInt/ITS4 primers. No amplified product was found from the other 16 isolates with cylindrical conidia, five isolates with fusiform and two isolates with falcate conidia.

\section{Phylogenetic analysis}

Phylogeny based on the ITS region. The ITS dataset contained 544 characters including alignment gaps, of which 139 characters were found parsimonyinformative. After sequencing of the ITS region of all 56 isolates, we discovered that there were several isolates with $100 \%$ identical sequences, representing 31 unique sequence types. Therefore, we only included 31 isolates in the phylogenetic study, i.e. one representative of each sequence type (Table 1). Six clades were generated from the analysis with high bootstrap support (Fig. 3). Eight Vietnamese representative isolates out of 20 having cylindrical conidia fell in the clade of C. gloeosporioides reference isolate (231) and together with the CBD reference isolate (C. kahawae, IMI357056) (Clade3) with 100\% support. This included 2 Vietnamese isolates that were unable to utilize tartrate. Five isolates with fusiform conidia were placed 
together with C. acutatum reference isolates (NI90 and 397). Clade 1 containing two isolates with falcate conidia grouped together with C. capsici (IMI 56173) with high support. Four isolates with cylindrical conidia, including isolates 606b (1) and BMT10 (L2) that were not able to metabolize ammonium tartrate grouped together with the reference isolates of $C$. boninense. The other isolates with cylindrical broadsized conidia and dark colony color fell into two separate clades (Clade 3 and clade 6) and were therefore considered as unknown species.

Phylogeny based on the mtSSU region. Similarly, the same groups of isolates as in the case of the ITS data set had identical sequences, thus we used the same subset of taxa as for constructing the ITS trees. The size of the mtSSU region varied substantially among the studied species: 407bp for C. gloeosporioides, 481bp for $C$. acutatum, 407bp for C. capsici, 412bp for C. boninense and 406 to 409bp for the isolates of unknown species. Out of more than 657 total characters, 122 were found parsimony-informative. The topology of the mtSSU tree was concordant with the ITS tree. Four main clades of C. gloeosporioides, C. acutatum, C. capsici and C. boninense were delimited with high bootstrap values (Fig. 4). All representative isolates of Vietnamese C. gloeosporioides and the reference of CBD (C. kahawae) were placed together in the same group as the reference C. gloeosporioides isolate 231 with 100\% support. Three clades were created from isolates of the unknown species and well supported by high bootstrap, which was higher than in the ITS tree. Clades 3a and 3b (Fig. 4) derived from subclades within clade 3 of the ITS tree. 
Isolates that were unable to use ammonium tartrate fell into three different groups of Colletotrichum species similar as in the ITS tree.

\section{Pathogenicity tests}

The CBD isolate (IMI 357057, C. kahawae) was the most pathogenic indicated by the high infection percentage (100\%) of both wounded and non-wounded green berries (Table 3). Conversely, most of the Vietnamese Colletotrichum isolates caused only moderate damage, mainly on wounded berries, with less severe and less sunken lesions than those caused by the reference CBD isolate (C. kahawae). However, several of the Vietnamese isolates also produced clear disease symptoms on nonwounded green berries although at a lower rate. The variability of the results was high as indicated by high SD values. A higher percent infection was found on wounded berries compared to non-wounded berries ( $P=0.0037$ and $P=0.0001$ for inoculation with agar plug and conidia, respectively). Six of the isolates belonging to $C$. gloeosporioides and one from unknown species were able to induce lesions on nonwounded berries. C. gloeosporioides isolate 119b (1) was the most pathogenic among the Vietnamese isolates, and it produced the same size of slightly sunken lesion as the CBD isolate but at lower rate. None of the isolates of $C$. acutatum, C. capsici or $C$. boninense produced symptoms on non-wounded berries. The percent infected berries differed significantly between C. gloeosporioides and the other species found in Vietnam ( $\mathrm{P}=0.0001$ and 0.014 for inoculation with agar plug and conidia, 
respectively). The repeated experiment showed the same results as the previous experiment. 


\section{Discussion}

By combining morphological characterization, species specific primers and sequencing analysis of the portion of mtSSU and ITS region we identified $C$. gloeosporioides, C. acutatum, C. capsici, and C. boninense as being associated with anthracnose disease on Coffea spp. in Vietnam. In addition, we found ten Colletotrichum isolates of unknown species. In total more than 30 isolates of those morphological types were found. Several of the Vietnamese isolates were found to be pathogenic, i.e. they produced lesions on detached green berries after inoculation.

Vietnamese $C$. acutatum species derived from coffee could be distinguished from $C$. gloeosporioides by using species-specific primers designated for either C. acutatum or C. gloeosporioides and by the phylogenetic analysis. C. acutatum was reported to be associated with disease on coffee in Papua New Guinea (Kenny et al. 2006). C. acutatum is a common and quite an aggressive pathogen on a variety of crops in tropical regions. However, on coffee plants in Africa Masaba and Waller (1992) have regarded this species as mainly saprophytic. We found no symptoms caused by this species on non-wounded berries, thus confirming observations of Massaba and Waller.

The majority of the Vietnamese isolates belonged to C. gloeosporioides and was found mainly on berries and not so frequent on twigs and leaves. All isolates of $C$. gloeosporioides and the reference isolate of $C$. kahawae fell in the main group of $C$. 
gloeosporioides based on both ITS and mtSSU sequence analyses. This is in agreement with earlier studies and indicates that $C$. kahawae should probably be considered as synonymous to C. gloeosporioides (Sreenivasaprasad et al. 1993; Cannon et al. 2008). However, there have been strong debates on taxonomic relevance of these taxa, e.g. Waller et al. (1993) stated that C. kahawae should be considered as a distinct species due to its high virulence, biochemical features and endemicity to Africa. In a recent study we reported high genetic variation in Vietnamese C. gloeosporioides populations causing anthracnose disease of coffee berries, based on RAPD and MpPCR markers (Nguyen et al. 2009). This is typical for C. gloeosporioides populations on different hosts (Mills et al. 1992; Abang et al. 2006), while studies of the genetic diversity within populations of the CBD-fungus (C. kahawae) revealed low diversity (Derso \& Waller. 2003; Bridge et al. 2008). Inability of C. kahawae to utilize ammonium tartrate as a sole carbon source can be a distinguishable criterion to differentiate C. kahawae from other Colletotrichum species associated with coffee berry disease (Waller et al. 1993; Bridge et al. 2008). Furthermore, Bridge et al. (2008) considered C. kahawae as a distinct taxon that can be separated from C. gloeosporioides by assessing mtDNA RFLP and VNTR. A significant difference in growth rate between C. gloeosporioides and C. kahawae was found at 25 and $30^{\circ} \mathrm{C}$ in our study. This correlated to previous reports by Waller et al. (1993) and Varzea et al. (2002). Two of the Vietnamese C. gloeosporioides isolates could not metabolize tartrate, but grew fast at high temperature. Pathogenicity tests on berries and hypocotyls can separate C. kahawae from other species of Colletotrichum (Varzea et al. 2002). According to our results, the Vietnamese C. 
gloeosporioides could be distinguished from C. kahawae on basis of growth rate, tartrate utilization and pathogenicity. C. kahawae caused deeply sunken lesions on the berry surface, then invaded and destroyed the bean similar to previous studies by Chen et al. (2005) and Masaba and Waller (1992), while Vietnamese C. gloeosporioides caused less severe disease symptoms.

Some isolates having somewhat larger cylindrical conidia than C. gloeosporioides, were identified as C. boninense (Moriwaki et al. 2003). This species has originally been isolated in Japan and has been described as a new species in the $C$. gloeosporioides complex (Moriwaki et al. 2003). Thereafter, it has been reported from South America, China, Australia, Zimbabwe and Brazil (Lu et al. 2004; Lubbe et al. 2004; Farr et al. 2006; Tozze Jr et al. 2009). C. boninense has been found on a broad range of hosts, i.e. mono/dicotyledonous, herbaceous and woody plants (Farr et al. 2006). We found the teleomorph in pure cultures in almost all Vietnamese $C$. boninense isolates, similar to $C$. boninense isolates derived from other host plants reported by Lu et al. (2004). To our knowledge, presence of teleomorph stage has never been reported in other studies of $C$. boninense. Pathogenicity of this species has not been studied except a short note about virulent ability of the species on Capsicum annuum by Tozze Jr et al. (2009). We found only slight symptoms on wounded berries and therefore this species may be regarded as a saprophyte.

Colletotrichum isolates with falcate conidia were identified as C. capsici. However, the taxonomic difference between C. capsici and C. dematium seems to be unclear, 
partly due to the lack of holotype material of species with falcate conidia. Lubbe et al. (2004) reported that C. capsici grouped together with C. dematium according to sequence analysis of either the ITS region or partial $\beta$-tubilin gene. C. capsici is reported to be a pathogenic fungus on a wide range of host plants in the tropics and subtropics (Sutton. 1980; 1992; Than et al. 2008). In contrast, C. dematium has been described as a saprophytic species (Mordue. 1971) and has mainly been found in temperature regions (Sutton. 1980). However, C. dematium has been designated as the same taxon as C. capsici (von Arx. 1957). Isolates with morphological characters as C. capsici, has earlier been found on infected coffee in Vietnam (Tran et al. 1998). The ITS sequences of our isolates showed $99 \%$ identity to the C. capsici strain (Ccmj7) associated with anthracnose disease on Capsicum spp. previously described by Than et al. (2008). They were not pathogenic due to lack of symptoms on nonwounded berries.

Apart from the Colletotrichum species described above, we found several Colletotrichum isolates having relatively massive, cylindrical conidia, abundant sclerotia and dark color in old cultures. These isolates were grouped into clades distinctly different from the other Colletotrichum species and therefore we considered them as unknown species. The cultural and morphological features of those isolates were considerably different from C. gloeosporioides and other Colletotrichum species described by Sutton (1980; 1992). Interestingly, BLAST search against the GenBank nucleotide database has resulted in close hits $(99 \%$ similarity and E-values $=0)$ to strains of endophytes of non-identified species of Colletotrichum from tropical plants 
like Coffea spp., Musa spp., Orchis spp. etc from previous studies (AY438553 and AY442184, Lu et al (2004)) and (AY 26644404, Photita et al .(2005)).

Moreover, two other isolates of unknown species, LD33 (L1) and LD11 (L3), that comprised a separate clade in our phylogenetic trees, were slightly morphologically different from C. gloeosporioides. They had dark grey colony color with felt-like surface and almost no aerial mycelium. The ITS sequences of these isolates gave high BLAST scores to sequences deposited by Farr et al. (2006) representing strains of unknown species of Colletotrichum originating from orchid genera Cattleya and Dendrobium. Since one of these isolates caused disease on non-wounded berries whilst other isolates caused minor symptoms on wounded berries further pathogenicity tests should be carried.

The results of the pathogenicity tests on detached green berries indicated that some of the Vietnamese isolates were virulent. Six isolates of $C$. gloeosporioides and one of unknown species produced lesions on non-wounded berries. One C. gloeosporioides isolate, 119b (1) also induced symptoms on both hypocotyls and berries in previous study by Nguyen et al. (2009). However, the disease symptoms were less severe than those produced by the CBD-fungus (C. kahawae). The Vietnamese isolates from this study seldom infected the coffee beans, although they produced sunken lesions on the skin of the berries. Higher number of C. gloeosporioides isolates and other Colletotrichum species were able to infect wounded berries than non-wounded berries 
indicating that the cuticular wax layer may inhibit the infection process of the pathogens. Wounding makes the host more susceptible as has been demonstrated formerly (Than et al. 2008). Based on our pathogenicity tests we assume that the disease on coffee in Vietnam is mainly caused by C. gloeosporioides, but other species may also be involved to enhance infection process especially under nutrient deficiency, physiological stress or during seasons of over-bearing of berries. $C$. gloeosporioides has been found to be responsible for "dieback" disease of branches due to exceeding berries in fruiting period (Chen et al. 2003). One of the isolates of unknown species also infected non-wounded berries and therefore it would be in consideration to describe this putative species and characterize its pathogenic abilities further.

To our knowledge this is the first systematic study of Colletotrichum species on coffee in Vietnam. The high biodiversity under tropical climate conditions can be one of reasons for the presence of multiple species of Colletotrichum on coffee found in this study. Recently, there have been studies on Colletotrichum spp. associated with a single host plant, e.g. strawberry (Ureña-Padilla et al. 2002; Martinez-Culebras et al. 2003) and pepper (Than et al. 2008). Taxonomical clarification of the putative unknown species has to be taken in consideration. No evidence of the existence of CBD (C. kahawae), the main pathogenic fungus threatening coffee production in Africa, has been found so far in Vietnam. The results of this study can be valuable for 
disease management's practices and development of more resistant coffee varieties for sustainable coffee production in Vietnam.

\section{Acknowledgements}

The authors acknowledge the Swedish International Development Agency (Sida) for financial support. We are thankful to Dr Sreenivasaprasad for kindly supplying isolates NI 90, 397 and PR220 used in this study. We also thank Ms Therese Bengtsson, Ngat Thi Hoang and Ann-Charlotte Strömdahl for excellent technical assistances. We acknowledge Mrs. Loang Kim Tran, Tram Thi Bui and Ha Thanh Nguyen for valuable assistances during field samplings. 


\section{References}

Abang, M. M., Asiedu, R., Hoffmann, P., Wolf, G. A., Mignouna, H. D. \& Winter, S. (2006). Pathogenic and genetic variability among Colletotrichum gloeosporioides isolates from different yam hosts in the agroecological zones in Nigeria. Journal of Phytopathology, 154, 51-61.

Bock, K. R. (1956). Investigations on coffee berry disease-laboratory studies. East African agricultural and forestry journal, 22, 97-103.

Bridge, P. D., Waller, J. M., Davies, D. \& Buddie, A. G. (2008). Variability of Colletotrichum kahawae in relation to other Colletotrichum species from tropical perennial crops and the development of diagnostic techniques. Journal of Phytopathology, 156, 274-280.

Cannon, P. F., Buddie, A. G. \& Bridge, P. D. (2008). The typification of Colletotrichum gloeosporioides. Mycotaxon, 104, 189-204.

Chen, Z. J., Liang, J. \& Rodrigues, C. J. J. (2005). Colletotrichum gloeosporioides can overgrow Colletotrichum kahawae on green coffee berries first inoculated with C. kahawae. Biotechnology Letters, 27, 679-682.

Chen, Z. J., Ribeiro, A., Silva, M. C., et al. (2003). Heat shock-induced susceptibility of green coffee leaves and berries to Colletotrichum gloeosporioides and its association to PR and hsp70 gene expression. Physiological and Molecular Plant Pathology, 63, 181-190.

Derso, E. \& Waller, J. M. (2003). Variation among Colletotrichum isolates from diseased coffee berries in Ethiopia. Crop Protection, 22, 561-565.

Ewing, B. \& Green, P. (1998). Base-calling of automated sequencer traces using phred. II. Error probabilities. Genome Research, 8, 186-194.

Farr, D. F., Aime, M. C., Rossman, A. Y. \& Palm, M. E. (2006). Species of Colletotrichum on agavaceae. Mycological Research, 110, 1395-1408.

Freeman, S. (2000). Genetic diversity and host specificity of Colletotrichum species on various fruits. Colletotrichum: Host Specificity, Pathology, and Host-Pathogen Interaction, 131-144.

Kenny, M. K., Galea, V. J., Scott, P. T. \& Price, T. V. (2006). A comparison of Colletotrichum species associated with berry diseases of Coffea arabica L. Pest and disease incursion: risk, threats and management in Papua New Guinea.ACIAR Technical Reports, 62, 192-199.

Li, K. N., Rouse, D. I. \& German, T. L. (1994). PCR primers that allow intergeneric differentiation of ascomycetes and their application to Verticillium spp. Applied and Environmental Microbiology, 60, 4324-4331.

Lu, G., Cannon, P. F., Reid, A. \& Simmons, C. M. (2004). Diversity and molecular relationships of endophytic Colletotrichum isolates from the Iwokrama Forest Reserve, Guyana. Mycological Research, 108, 53-63.

Lubbe, C. M., Denman, S., Cannon, P. F., Groenewald, J. Z., Lamprecht, S. C. \& Crous, P. W. (2004). Characterization of Colletotrichum species associated with diseases of Proteaceae. Mycologia, 96, 1268-1279.

Martinez-Culebras, P. V., Querol, A., Suarez-Fernandez, M. B., Garcia-Lopez, M. D. \& Barrio, E. (2003). Phylogenetic relationships among Colletotrichum pathogens 
of strawberry and design of PCR primers for their identification. Journal of Phytopathology, 151, 135-143.

Masaba, D. M. \& Waller, J. M. (1992). Coffee berry disease: The current status. (In: J. A. Bailey \& M. J. Jeger, (Eds.), Colletotrichum: Biology, Pathology and Control (pp. 237-249). Wallingford UK: CAB International.)

Mills, P. R., Hodson, A. \& Brown, A. E. (1992). Molecular differentiation of Colletotrichum gloeosporioides isolates infecting tropical fruits. (In: J. A. Bailey \& M. J. Jeger, (Eds.), Colletotrichum- Biology, pathology and control (pp. 269288). CAB International Mycological Institute: UK.)

Mordue, J. E. M. (1971). CMI Descriptions of Pathogenic Fungi and Bacteria. (Kew: Commonwealth Mycological Institute)

Moriwaki, J., Sato, T. \& Tsukiboshi, T. (2003). Morphological and molecular characterization of Colletotrichum boninense sp. nov. from Japan. Mycoscience, 44, 47-53.

Nguyen, T. H. P., Säll, T., Bryngelsson, T. \& Liljeroth, E. (2009). Variation among Colletotrichum gloeosporioides isolates from infected coffee berries at different locations in Vietnam. Plant Pathology, Doi: 10.1111/j.1365-3059.2009.02085.x.

Photita, W., Taylor, P. W. J., Ford, R., Hyde, K. D. \& Lumyong, S. (2005). Morphological and molecular characterization of Colletotrichum species from herbaceous plants in Thailand. Fungal Diversity, 18, 117-133.

Pinkert, C. (2004). Nutrient \& quality analysis of coffee cherries in Huong Hoa district, Vietnam (Wageningen UR, The Netherlands: Plant research international B.V)

Smith, B. J. \& Black, L. L. (1990). Morphological, cultural, and pathogenic variation among Colletotrichum species isolated from strawberry. Plant Disease, 74, 6976.

Sreenivasaprasad, S., Brown, A. E. \& Mills, P. R. (1992). DNA sequence variation and interrelationships among Colletotrichum species causing strawberry anthracnose. Physiological and Molecular Plant Pathology, 41, 265-281.

Sreenivasaprasad, S., Brown, A. E. \& Mills, P. R. (1993). Coffee berry disease pathogen in Africa: genetic structure and relationship to the group species Colletotrichum gloeosporioides. Mycological Research, 97, 995-1000.

Sreenivasaprasad, S., Sharada, K., Brown, A. E. \& Mills, P. R. (1996). PCR-based detection of Colletotrichum acutatum on strawberry. Plant Pathology, 45, 650.

Sutton, B. C. (1980). The Coelomycetes. Fungi Imperfecti with Pycnidia, Acervuli and Stromata. (Kew: Commonwealth Mycological Institute)

Sutton, B. C. (1992). The genus Glomerella and its anamorph Colletotrichum. (In, Colletotrichum: biology, pathology and control (pp. 1-26). CAB International, Wallingford.)

Than, P. P., Jeewon, R., Hyde, K. D., Pongsupasamit, S., Mongkolporn, O. \& Taylor, P. W. J. (2008). Characterization and pathogenicity of Colletotrichum species associated with anthracnose on chilli (Capsicum spp.) in Thailand. Plant Pathology, 57, 562-572. 
Tozze Jr, H. J., Massola Jr, N. M., Camara, M. P. S., et al. (2009). First Report of Colletotrichum boninense Causing Anthracnose on Pepper in Brazil. Plant Disease, 93, 106-106.

Tran, K. L., Vu, T. T. N. \& Ngo, T. X. T. (1998). Results of investigation of coffee berry disease and die-back on Arabica coffee var. Catimor. Monthly Journal of Science, technology and economic management (in Vietnamese), 6, 253-255.

Ureña-Padilla, A. R., Mackenzie, S. J., Bowen, B. W. \& Legard, D. E. (2002). Etiology and population genetics of Colletotrichum spp. causing crown and fruit rot of strawberry. Phytopathology, 92, 1245-1252.

Varzea, V. M., Rodrigues Jr, C. J. \& Lewis, B. G. (2002). Distinguishing characteristics and vegetative compatibility of Colletotrichum kahawe in comparison with other related species from coffee. Plant Pathology, 51, 202.

Vinnere, O., Fatehi, J., Wright, S. A. I. \& Gerhardson, B. (2002). The causal agent of anthracnose of Rhododendron in Sweden and Latvia. Mycological Research, 106, 60-69.

Von Arx, J. A. (1957). Die arten der Gattung Colletotrichum cda. Phytopathologische Zeitschrift, 29, 413-468.

Waller, J. M. (1992). Colletotrichum diseases of perennial and other cash crops. (In: J. A. Bailey \& M. J. Jeger, (Eds.), Colletotrichum: Biology, Pathology and Control (pp. 131-142). Wallingford: CAB International.)

Waller, J. M., Bridge, P. D., Black, B. \& Hakiza, G. (1993). Characterization of the coffee berry disease pathogen Colletotrichum kahawae sp. nov. Mycological Research, 97, 989-994.

White, T. J., Bruns, T., Lee, S. \& Taylor, J. (1990). Amplification and direct sequencing of fungal ribosomal RNA genes for phylogenetics. PCR protocols: $a$ guide to methods and applications, 315, 322.

Zar, J. (1984). Biostatistical analysis. (Englewood Cliffs: Prentice-Hall International) 


\section{Legends to figures}

Figure1. Locations of coffee growing plantations where samples for Colletotrichum isolation were collected in Vietnam.

Figure 2: a. Average growth rate of isolates of C. gloeosporioides (Cg, 20 isolates), C. kahawae (CBD, 3 isolates) and isolates that could not use tartrate $(\mathrm{Cg}$, tartrate negative, 2 isolates). Bars represent standard deviation of the mean. b. Average growth rate of $C$. acutatum (Cacut), $C$. capsici (Ccapsi), $C$ boninense (Cbonin) and the three unknown species. Bar indicate mean standard deviation from ANOVA. Different letter indicate significant differences between group means $(\mathrm{P}<0.05)$ (Tukey’s studentized range test). For vertical comparison only.

Figure 3: Maximum Parsimony (MP) tree based on the ITS sequences of 31 sequence types including 24 Vietnamese Colletotrichum isolates and reference Colletotrichum species listed in Table 1 . The tree was rooted by F. redolens; 1000 bootstrap data sets and Kimura-2P model distance matrix were used; bootstrap values above $50 \%$ are shown on internal branches.

Figure 4: Maximum Pasimonry (MP) tree based on the mtSSU sequences of 31 sequence types including 24 Vietnamese Colletotrichum isolates and reference Colletotrichum species listed in Table 1 . The tree was rooted by F. redolens; 1000 bootstrap data sets and Kimura-2P model distance matrix were used; bootstrap values above $50 \%$ are shown on internal branches. 
Table 1. Origins of Colletotrichum spp isolated from coffee in Vietnam and Colletotrichum reference isolates

\begin{tabular}{|c|c|c|c|c|c|c|c|}
\hline \multirow[t]{3}{*}{ Name of isolate } & \multirow[t]{3}{*}{ Identified as } & \multirow[t]{3}{*}{ Location } & \multirow[t]{3}{*}{ Tissue $^{\mathrm{y}} /$ Host } & \multirow{2}{*}{\multicolumn{3}{|c|}{$\begin{array}{l}\text { GenBank accession } \\
\text { number of representative } \\
\text { isolates }\end{array}$}} & \multirow{3}{*}{$\begin{array}{l}\text { Tartrate } \\
\text { utilization }\end{array}$} \\
\hline & & & & & & & \\
\hline & & & & No. $^{z}$ & ITS & MtSSU & \\
\hline $\left.\operatorname{SL1a}{ }^{*}\right)^{*}$ & C. gloeosporioides & $\mathrm{N}$ & Coffee berries & 1 & FJ968590 & FJ968627 & + \\
\hline NA100(5) & C. gloeosporioides & ” & $”$ & 1 & & & + \\
\hline NA302 & C. gloeosporioides & ” & $”$ & 1 & & & + \\
\hline NA200a(3) & C. gloeosporioides & ” & ” & 1 & & & + \\
\hline SL2a(8) & C. gloeosporioides & ” & $”$ & 1 & & & + \\
\hline SL304a(1) & C. gloeosporioides & ” & $”$ & 1 & & & + \\
\hline SL207a(6) & C. gloeosporioides & ” & ” & 1 & & & + \\
\hline 213(2) & C. gloeosporioides & ” & $”$ & 1 & & & + \\
\hline NA40a(11) & C. gloeosporioides & ” & ” & 2 & FJ968589 & FJ968626 & - \\
\hline NA4a(9) & C. gloeosporioides & ” & $”$ & 2 & & & + \\
\hline LD43(B4) & C. gloeosporioides & $\mathrm{S}$ & $”$ & 2 & & & + \\
\hline LD11(B3) & C. gloeosporioides & $”$ & $”$ & 2 & & & + \\
\hline $606 b(4)^{*}$ & C. gloeosporioides & ” & $”$ & 3 & FJ968591 & FJ968628 & + \\
\hline $309 b(2)$ & C. gloeosporioides & $\mathrm{N}$ & $”$ & 3 & & & + \\
\hline 702(1) & C. gloeosporioides & S & $”$ & 3 & & & + \\
\hline $12 c(3)$ & C. gloeosporioides & $\mathrm{N}$ & $”$ & 3 & & & + \\
\hline $119 b(1)^{*}$ & C. gloeosporioides & ” & $”$ & 4 & FJ968596 & FJ968631 & + \\
\hline 201(1) & C. gloeosporioides & $”$ & $”$ & 4 & & & + \\
\hline $803 b(1)^{*}$ & C. gloeosporioides & ” & $”$ & 5 & FJ968592 & FJ968629 & + \\
\hline
\end{tabular}




\begin{tabular}{|c|c|c|c|c|c|c|c|}
\hline 531(7) & C. gloeosporioides & $”$ & $"$ & 5 & & & + \\
\hline $478 a(3)^{*}$ & C. gloeosporioides & S & $"$ & 6 & FJ968595 & FJ968630 & + \\
\hline BMT16(T3)* & C. gloeosporioides & $”$ & Coffee twigs & 7 & FJ968593 & FJ968632 & - \\
\hline LD46a(L1) ${ }^{*}$ & C. gloeosporioides & $"$ & Coffee leaves & 8 & FJ968594 & FJ968625 & + \\
\hline SL204a* & C. capsici & $\mathrm{N}$ & Coffee berries & 9 & FJ968578 & FJ968606 & + \\
\hline BV14* & C. capsici & $"$ & $"$ & 10 & FJ968579 & FJ968607 & + \\
\hline $397^{* \dagger}$ & C. acutatum & USA & $\begin{array}{l}\text { Fragaria } \quad x \\
\text { ananassa }\end{array}$ & 11 & AF411734 & AF411749 & n.t \\
\hline ВMT26(L1) & C. acutatum & S & Coffee leaves & 11 & & & + \\
\hline BMT26(T2) & C. acutatum & $"$ & Coffee twigs & 11 & & & + \\
\hline 413(1) & C. acutatum & $\mathrm{S}$ & Coffee berries & 11 & & & + \\
\hline $213(1)^{*}$ & C. acutatum & $\mathrm{N}$ & $”$ & 12 & FJ968598 & FJ968609 & + \\
\hline BMT(HL)19* & C. acutatum & S & Coffee leaves & 13 & FJ968601 & FJ968610 & + \\
\hline $606 \mathrm{~b}(1)^{*}$ & C. boninense & $"$ & Coffee berries & 14 & FJ968580 & FJ968611 & - \\
\hline LD35a(T2) ${ }^{*}$ & C. boninense & $"$ & Coffee twigs & 15 & FJ968582 & FJ968616 & + \\
\hline LD8(L3)* & C. boninense & $"$ & Coffee leaves & 16 & FJ968583 & FJ968617 & + \\
\hline BMT10(L2)* & C. boninense & $"$ & $”$ & 17 & FJ968581 & FJ968615 & - \\
\hline LD35b(L1) & C. boninense & $"$ & $"$ & 17 & & & + \\
\hline LD43(T4) & C. boninense & $"$ & Coffee twigs & 17 & & & + \\
\hline LD35b(B2) ${ }^{*}$ & Unknown species ${ }^{\mathrm{x}}$ & $"$ & Coffee berries & 18 & FJ968586 & FJ968619 & + \\
\hline LD27(T1) & Unknown species ${ }^{\mathrm{x}}$ & $"$ & Coffee twigs & 18 & & & + \\
\hline LD35b(T1) & Unknown species ${ }^{x}$ & $"$ & $"$ & 18 & & & + \\
\hline BMT10(T2)* & Unknown species ${ }^{\mathrm{x}}$ & $"$ & $"$ & 19 & FJ968588 & FJ968618 & + \\
\hline LD30a(T3) & Unknown species ${ }^{\mathrm{x}}$ & $"$ & $"$ & 19 & & & + \\
\hline LD30a(T4) ${ }^{*}$ & Unknown species ${ }^{\mathrm{x}}$ & $”$ & $"$ & 20 & FJ968587 & FJ968622 & - \\
\hline BMT25(L3)* & Unknown species $^{\mathrm{v}}$ & $”$ & Coffee leaves & 21 & FJ968585 & FJ968620 & + \\
\hline LD16(L2) ${ }^{*}$ & Unknown species $^{\mathrm{v}}$ & $”$ & $”$ & 22 & FJ968584 & FJ968621 & + \\
\hline
\end{tabular}




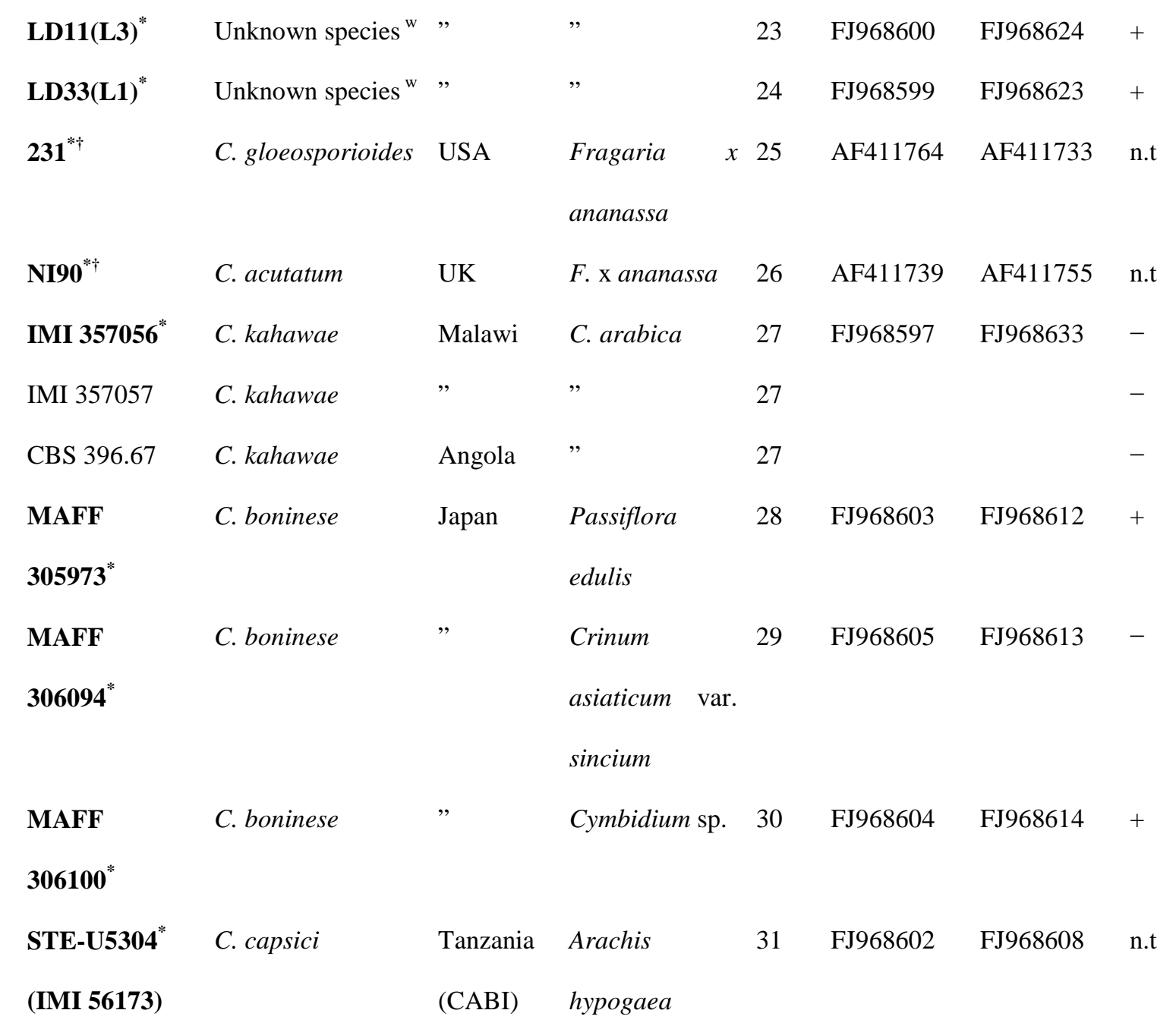

N: North; S: South of Vietnam.

+: positive in amonium tatrate utilization; -: negative in amonium tatrate utilization; n.t.: not tested.

${ }^{\dagger}$ reference isolates sequenced by Vinnere et al. (2002).

v, w, and x isolates belong to unknown species of Colletotrichum; group v, w , and x, respectively.

${ }^{\mathrm{y}}$ isolated from Coffea arabica, except isolates 606b (1) and 606b (4) isolated from C. robusta.

${ }^{z}$ Sequence group No. 1-31. All isolates of each sequence group had identical sequences. Therefore, only one of them was included in the phylogenetic analysis; indicated by * and bold letters. 
Table 2: Conidial measurements of Colletotrichum species originated from diseased coffee in Vietnam

\begin{tabular}{|c|c|c|c|c|}
\hline Species & $\begin{array}{l}\text { Number of } \\
\text { isolates }\end{array}$ & $\begin{array}{l}\text { Conidial length, width }(\mu \mathrm{m}) \\
\text { (conidial shape) }\end{array}$ & $\begin{array}{l}\text { Mean length } \\
(\mu \mathrm{m})\end{array}$ & $\begin{array}{l}\text { Mean width }^{\mathrm{a}} \\
(\mu \mathrm{m})\end{array}$ \\
\hline C. gloeosporioides & 23 & 9.6-20.8 x 2.9-8,7 (Cylindrical ) & $15.0 \mathrm{c}$ & 4.2 cde \\
\hline C. acutatum & 5 & 7.9-19.2 x 2.5-6.2 (Fusiform) & $12.8 \mathrm{c}$ & 4.0 de \\
\hline C. capsici & 2 & 15.3-28.0 x 2.5-3.6 (Falcate) & $22.5 \mathrm{~b}$ & $3.1 \mathrm{e}$ \\
\hline C. boninese & 6 & 11.8-25.2 x 4.4-7.1 (Cylindrical ) & $14.7 \mathrm{c}$ & $5.8 \mathrm{bc}$ \\
\hline Unknown species ${ }^{\mathrm{v}}$ & 2 & 25.4-40.6x 8.4-11.7 (Cylindrical ) & $32.1 \mathrm{a}$ & $10.2 \mathrm{a}$ \\
\hline Unknown species ${ }^{\mathrm{w}}$ & 2 & 13.6-17.3 x 4.7-6.2 (Cylindrical ) & $15.3 \mathrm{c}$ & $4.8 \mathrm{~cd}$ \\
\hline Unknown species ${ }^{\mathrm{x}}$ & 6 & 14.8-30.2 x 5.6-8.8 (Cylindrical ) & $21.2 \mathrm{~b}$ & $7.11 \mathrm{~b}$ \\
\hline
\end{tabular}

a Different letters indicate significant differences. Tukey's multiple range test, $\mathrm{p}<0.05$. For vertical comparison only. 
Table 3: Pathogenicy tests of Colletotrichum species on wounded and non-wounded detached green berries of coffee

\begin{tabular}{|c|c|c|c|c|c|c|c|c|}
\hline \multirow{5}{*}{$\begin{array}{l}\text { Name } \\
\text { isolates }\end{array}$} & \multirow[t]{5}{*}{ Identified as } & \multirow{5}{*}{$\begin{array}{l}\text { Agar plug } \\
\text { (A) or } \\
\text { Conidia } \\
\text { (C) }\end{array}$} & \multirow{3}{*}{$\begin{array}{l}\% \text { infected } \\
\text { lesion }^{\mathrm{f}} \\
\text { Means } \pm S D\end{array}$} & \multirow[t]{3}{*}{ berries with } & \multicolumn{4}{|c|}{ Lesion diameter $(\mathrm{mm})^{\mathrm{g}}$} \\
\hline & & & & & \multicolumn{4}{|l|}{ Mean \pm SD } \\
\hline & & & & & & & & \\
\hline & & & Wounded & Non- & Wounded & $\mathrm{N}$ & Non- & $\mathrm{N}$ \\
\hline & & & & wounded & & & wounded & \\
\hline NA4a(9) & C. gloeosporioides & $\mathrm{C}$ & $30 \pm 20$ & $3 \pm 6$ & $11.1 \pm 4.7$ & 9 & 6.0 & 1 \\
\hline SL2a(8) & C. gloeosporioides & $\mathrm{C}$ & $20 \pm 17$ & 0 & $5.7 \pm 3.2$ & 6 & - & 0 \\
\hline SL1a(9) & C. gloeosporioides & A & $7 \pm 6$ & $13 \pm 12$ & $6.5 \pm 0.7$ & 2 & $8.5 \pm 4.4$ & 4 \\
\hline 119b(1) & C. gloeosporioides & A & $47 \pm 15$ & $20 \pm 10$ & $12.1 \pm 3.6$ & 14 & $11.8 \pm 2.7$ & 6 \\
\hline 201(1) & C. gloeosporioides & A & $3 \pm 6$ & 0 & 10 & 1 & - & 0 \\
\hline 531(7) & C. gloeosporioides & A & $10 \pm 10$ & 0 & $6.3 \pm 3.2$ & 3 & - & 0 \\
\hline $309 b(2)$ & C. gloeosporioides & C & 0 & 0 & - & 0 & - & 0 \\
\hline $702(1)$ & C. gloeosporioides & A & $23 \pm 32$ & 0 & $10.9 \pm 3.2$ & 7 & - & 0 \\
\hline $606 b(4)$ & C. gloeosporioides & $\mathrm{C}$ & $17 \pm 6$ & $3 \pm 6$ & $10.2 \pm 4.0$ & 5 & 4.0 & 1 \\
\hline 803b(1) & C. gloeosporioides & $\mathrm{C}$ & $23 \pm 15$ & $7 \pm 12$ & $10.4 \pm 4.9$ & 7 & $6.0 \pm 0.0$ & 2 \\
\hline $12 \mathrm{c}(3)$ & C. gloeosporioides & A & $7 \pm 12$ & $7 \pm 6$ & $10.0 \pm 0.0$ & 2 & $6.0 \pm 0.0$ & 2 \\
\hline SL204a & C. capsici & A & $3 \pm 6$ & 0 & 6.0 & 1 & - & 0 \\
\hline BV14 & C. capsici & $\mathrm{C}$ & $10 \pm 0$ & 0 & $7.3 \pm 2.5$ & 3 & - & 0 \\
\hline 413(1) & C. acutatum & $\mathrm{C}$ & $20 \pm 10$ & 0 & $10.7 \pm 4.1$ & 6 & - & 0 \\
\hline 213(1) & C.acutatum & $\mathrm{C}$ & $7 \pm 6$ & 0 & $6.0 \pm 0.0$ & 2 & - & 0 \\
\hline BMT26(L1) & C. acutatum & $\mathrm{C}$ & $13 \pm 15$ & 0 & $5.5 \pm 1.0$ & 4 & - & 0 \\
\hline BMT26(T2) & C. acutatum & C & $3 \pm 6$ & 0 & 6.0 & 1 & - & 0 \\
\hline BMT(HL)19 & C. acutatum & C & $13 \pm 12$ & 0 & $9.5 \pm 4.8$ & 4 & - & 0 \\
\hline
\end{tabular}




\begin{tabular}{|c|c|c|c|c|c|c|c|c|}
\hline LD35a(T2) & C. boninense & $\mathrm{C}$ & $7 \pm 6$ & 0 & $8.5 \pm 9.2$ & 2 & - & 0 \\
\hline LD35b(L1) & C. boninense & $\mathrm{C}$ & $23 \pm 6$ & 0 & $8.6 \pm 4.1$ & 7 & - & 0 \\
\hline BMT10(L2) & C. boninense & A & 0 & 0 & - & 0 & - & 0 \\
\hline LD16(L2) & Unknown species $^{v}$ & A & $3 \pm 6$ & 0 & 2.0 & 1 & - & 0 \\
\hline LD33(L1) & Unknown species ${ }^{\mathrm{w}}$ & $\mathrm{C}$ & $3 \pm 6$ & 0 & 14.0 & 1 & - & 0 \\
\hline LD27(T1) & Unknown species ${ }^{\mathrm{x}}$ & A & 0 & 0 & - & 0 & - & 0 \\
\hline LD35b(B2) & Unknown species ${ }^{\mathrm{x}}$ & A & $3 \pm 6$ & 0 & 6.0 & 1 & - & 0 \\
\hline LD30a(T4) & Unknown species ${ }^{x}$ & A & $13 \pm 6$ & $7 \pm 12$ & $6.5 \pm 1.0$ & 4 & $8.0 \pm 2.8$ & 2 \\
\hline LD30a(T3) & Unknown species ${ }^{x}$ & A & $3 \pm 6$ & 0 & 5.0 & 1 & - & 0 \\
\hline BMT25(L3) & Unknown species ${ }^{\mathrm{v}}$ & A & 0 & 0 & - & 0 & - & 0 \\
\hline IMI 357057 & C. kahawae & $\mathrm{C}$ & $100 \pm 0$ & $100 \pm 0$ & $10.5 \pm 3.4$ & 30 & $9.7 \pm 3.3$ & 30 \\
\hline
\end{tabular}

-: No lesion

$\mathrm{N}$ : Number of infected berries among 30 tested green berries

${ }^{\mathrm{e}}$ Inoculation method: agar plugs (A) or conidial suspension (C).

${ }^{\mathrm{f}}$ Infected berries were only counted if the lesions were developed from the inoculation point

${ }^{\mathrm{g}}$ Diameter of lesions on the same infected berries 
Figure 1
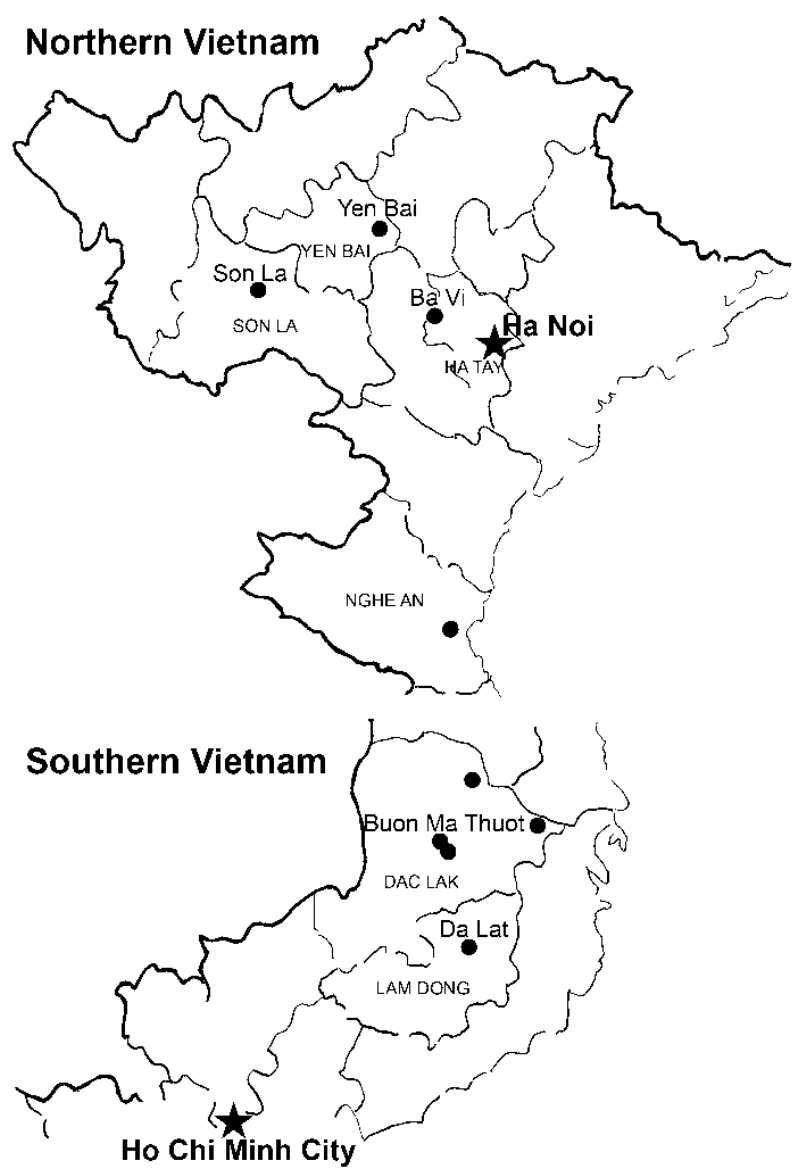
Figure 2
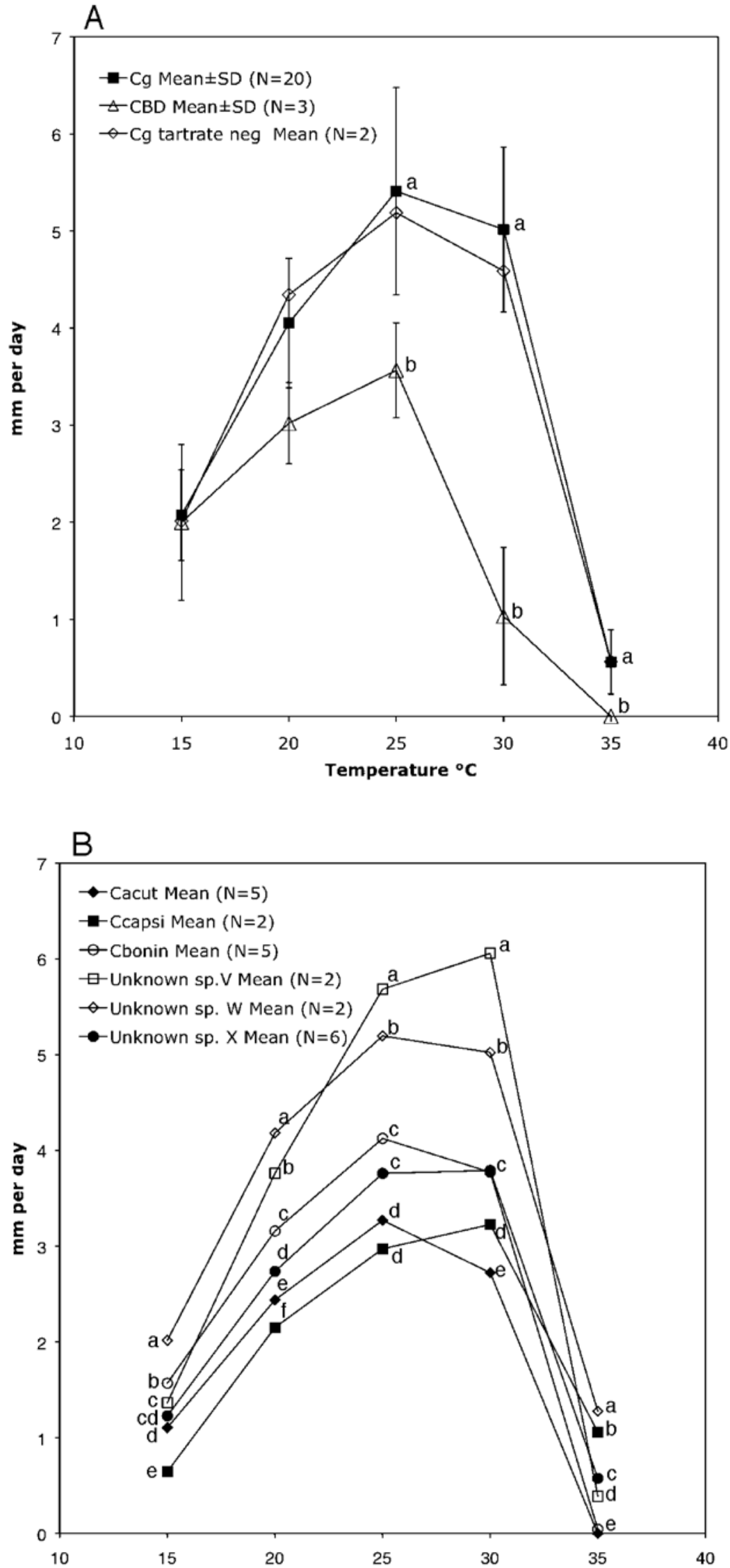
Figure 3

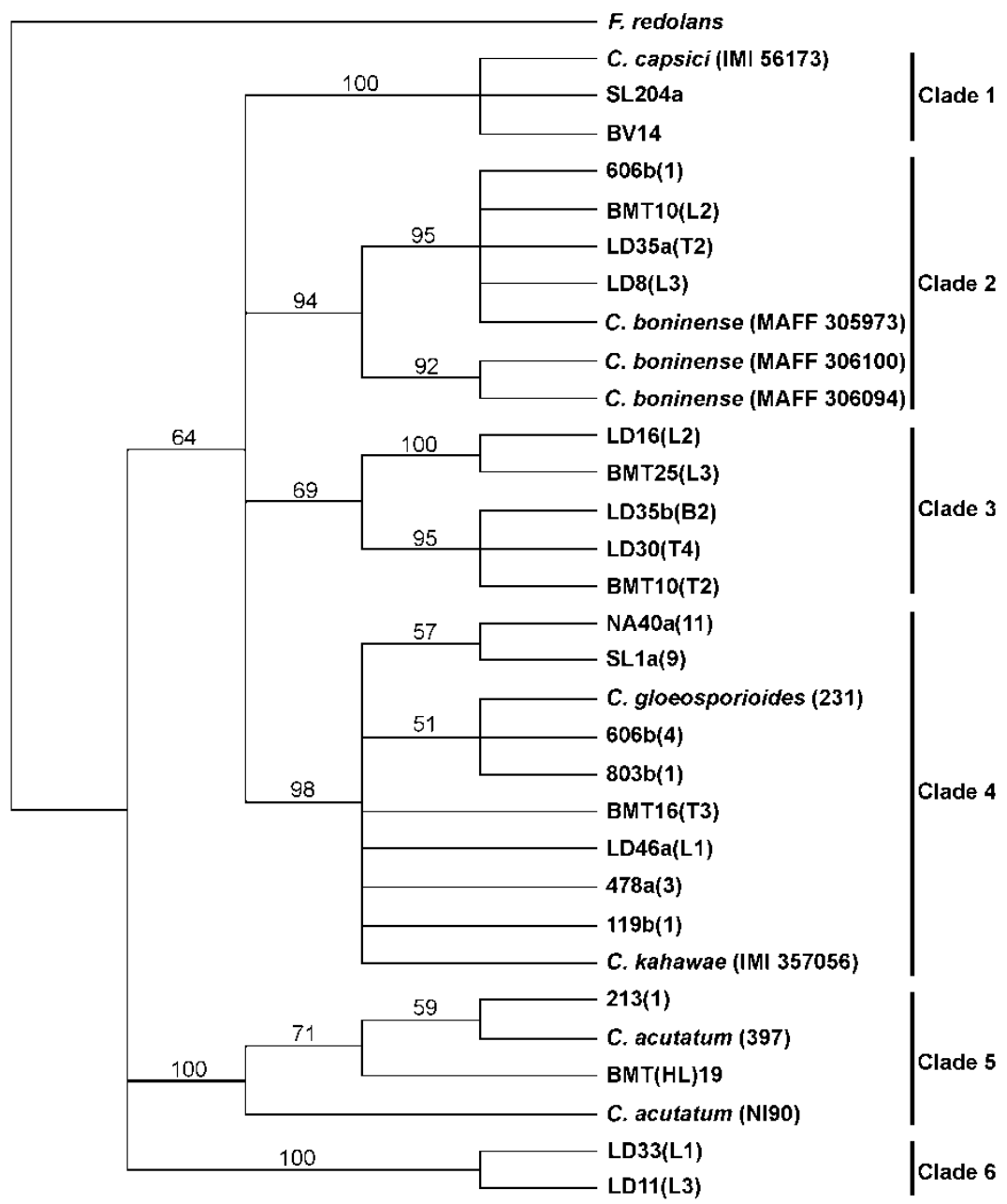


Figure 4

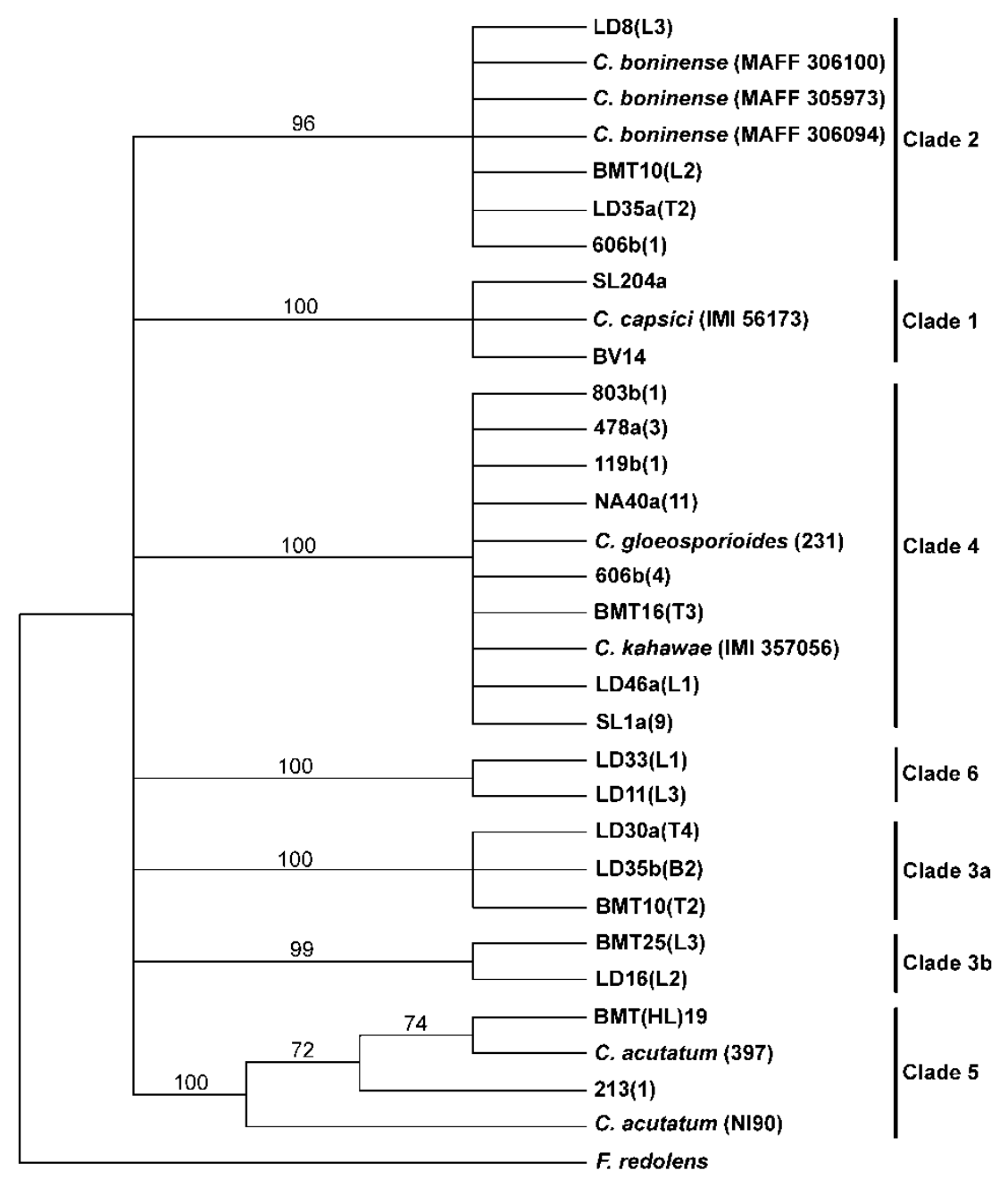

